\title{
Sinterização de cerâmicas em microondas. Parte I: Aspectos fundamentais
}

\section{(Microwave sintering of ceramics. Part I: Fundamental aspects)}

\author{
R. R. Menezes, P. M. Souto, R. H. G. A. Kiminami \\ Laboratório de Desenvolvimento e Processamento de Materiais em Microondas - LaDProM \\ Departamento de Engenharia de Materiais, Universidade Federal de S. Carlos \\ Rod. Washington Luiz, km 235, S. Carlos, SP 13565-905 \\ rrmboca@iris.ufscar.br,ruth@power.ufscar.br
}

\begin{abstract}
Resumo
O processamento de materiais baseado no aquecimento por meio de energia de microondas vem ganhado a cada dia mais destaque e importância em várias aplicações industriais, em virtude de uma série de vantagens em potenciais frente aos métodos convencionais de aquecimento. Na sinterização de materiais cerâmicos o uso de microondas permite redução no tempo de processamento, economia de energia e melhora na uniformidade microestrutural dos corpos cerâmicos. Como conseqüência das vantagens frente às técnicas de aquecimentos convencionais, a sinterização usando microondas vem sendo estudada por vários grupos de pesquisa em todo o mundo. Entretanto, os benefícios do uso das microondas só são obtidos quando a sinterização é realizada com o controle e entendimento científico de uma série de parâmetros e aspectos envolvidos no processo. Assim, esse trabalho tem por objetivo abordar aspectos científicos fundamentais do processo de sinterização de cerâmicas usando microondas, enfocando particularmente a interação matéria/microondas, aspectos peculiares do processo e a técnica de sinterização híbrida.

Palavras-chave: microondas, sinterização, cerâmicas.
\end{abstract}

\section{Abstract}

Processing of materials based on heating by microwave energy has gained increasing importance in many industrial applications due to its potential advantages over conventional heating methods. In the sintering of ceramic materials, the use of microwave energy decreases the processing time, saves energy and improves the microstructural homogeneity of ceramic bodies. These advantages have motivated various research groups around the world to study microwave sintering. However, the benefits deriving from the use of microwaves depend on the control and scientific understanding of the parameters and aspects of the process. This paper offers a review of the fundamental scientific aspects of microwave sintering of ceramics, focusing on the interaction between materials and microwaves and highlighting particular points of the hybrid sintering process and methodology.

Keywords: microwaves, sintering, ceramics.

\section{INTRODUÇÃO}

A energia de microondas é usada há mais de 40 anos no processamento de materiais, em aplicações que vão desde o aquecimento para reticulação de borrachas e cura de resinas a processos de secagem de cerâmicas e imobilização de resíduos.

Vários são os benefícios do uso da energia de microondas, como redução nos tempos de processamento e economia de energia. $\mathrm{O}$ que faz que vários processos baseados no aquecimento por microondas encontrem aplicações industriais. O principal benefício da exploração do uso da energia de microondas em processos ativados termicamente vem da possibilidade de especificidade de absorção da energia de microondas e aquecimento volumétrico dos materiais, em contraste com os métodos de aquecimento comumente utilizados. A energia de microondas transformase em calor dentro do material, o que resulta, em regra geral, em significante economia de energia e redução do tempo de processamento, o que vem mostrando ser o fator decisivo na aceitação do uso das microondas em muitas aplicações industriais.
Enquanto que a maioria das aplicações comerciais da energia de microondas esta voltada para processos que utilizam temperaturas relativamente baixas, como nas áreas de alimentos, madeira, borracha, polímeros e secagem de cerâmicas, há um crescente interesse no processamento de materiais por microondas em altas temperaturas.

O recente interesse no processamento de materiais é evidenciado pelo grande número de simpósios e congressos que vêm sendo dedicados ao processamento de materiais utilizando energia de microondas. A Sociedade de Pesquisa em Materiais (MRS - Materials Research Society), a Sociedade Americana de Cerâmica (American Ceramic Society), o Grupo de Pesquisa em Microondas (Microwave Working Group), a Associação Européia para Pesquisa e Ensino em Energia de Microondas (Association for Microwave Power in Europe for Research and Education), o Instituto Internacional de Energia de Microondas (International Microwave Power Institute), o Instituto de Aplicação de Ondas Eletromagnéticas (Institute of Electromagnetic Wave Application), o Instituto de Engenheiros da Austrália (Institution of Engineers, Australia) entre outros, vêm realizando vários congressos e simpósios focados no 
processamento de materiais, abordando desde as muitas dificuldades associadas ao processamento de materiais em microondas às recentes pesquisas em desenvolvimento de equipamentos.

Nesse sentido, o processamento de cerâmicas ocupa uma posição de destaque, em virtude dos vários estudos que vêm sendo desenvolvidos desde a década de 60. Esses estudos evidenciaram uma série de peculiaridades do uso da energia de microondas no processamento em altas temperaturas de materiais cerâmicos, como redução no consumo de energia e no tempo de processamento, distribuição invertida do perfil de temperatura e aquecimento seletivo.

Em cerâmicas, as microondas podem ser usadas para sinterização, síntese, secagem ou eliminação de orgânicos. Apesar da energia de microondas vir sendo usada com sucesso para sinterizar uma larga variedade de cerâmicas, sejam óxidos ou não óxidos, compósitos ou vidros, os requisitos particulares da sinterização de cerâmicas torna esse processo um dos maiores desafios de aplicação do processamento usando microondas. Esses requisitos freqüentemente incluem alguns ou todos dos seguintes pontos: elevadas temperaturas, elevadas taxas de aquecimento, uniformidade de temperatura e história térmica equivalente em toda a amostra. Buscando sinterizar materiais cerâmicos pelo aquecimento direto com microondas, alguns problemas fundamentais são geralmente encontrados. Primeiramente, a característica de pequena absorção de microondas de muitas cerâmicas em baixas temperaturas torna o aquecimento inicial muito difícil. Segundo, instabilidades térmicas podem ocorrer, o que pode levar ao fenômeno de "thermal runaway" descontrolado e a amostra superaquecer catastroficamente. Isto ocorre para uma variedade de materiais cerâmicos, incluindo, $\mathrm{Al}_{2} \mathrm{O}_{3}$, $\mathrm{SiO}_{2}, \mathrm{Fe}_{2} \mathrm{O}_{3}, \mathrm{ZrO}_{2}, \mathrm{ZnO}$, etc. Finalmente, o gradiente térmico característico do processo de aquecimento volumétrico que pode levar à heterogeneidades severas de temperatura. Em muitos experimentos de sinterizações o isolamento tem sido usado para minimizar esses gradientes. Todavia, o uso de isolamento pode agravar seriamente o fenômeno de "thermal runaway", o que gerou o desenvolvimento das técnicas de sinterização híbrida.

Observa-se, então, que a sinterização de cerâmicas utilizando-se energia de microondas é um processo que apresenta uma enorme quantidade de benefícios em potenciais, que, entretanto, só podem ser obtidos com o controle e entendimento de uma série de pontos e questões particulares e característicos do processamento de cerâmicas com o uso de microondas. Assim, esse trabalho tem por objetivo abordar aspectos fundamentais do processo de sinterização de cerâmicas usando energia de microondas, enfocando a interação matéria/microondas, aspectos peculiares do processo, como o "thermal runaway" e o processo de sinterização híbrida.

\section{PROCESSAMENTO POR MICROONDAS}

Nas últimas décadas, fornos de microondas tornaram-se um acessório comum na maioria das cozinhas. Rápidas taxas de aquecimento e economia de energia sobre os métodos convencionais de cozimento são os principais benefícios que trouxeram às cozinhas modernas. No entanto, apesar da utilização das microondas para o cozimento de alimentos ter se disseminado por toda a sociedade, a aplicação dessa tecnologia no processamento eficiente de materiais é um desenvolvimento relativamente novo. $\mathrm{O}$ uso de energia de microondas para o processamento de materiais tem o potencial de oferecer vantagens similares à sua utilização para cozimento de alimentos, como a redução no tempo de processamento e economia de energia [1].
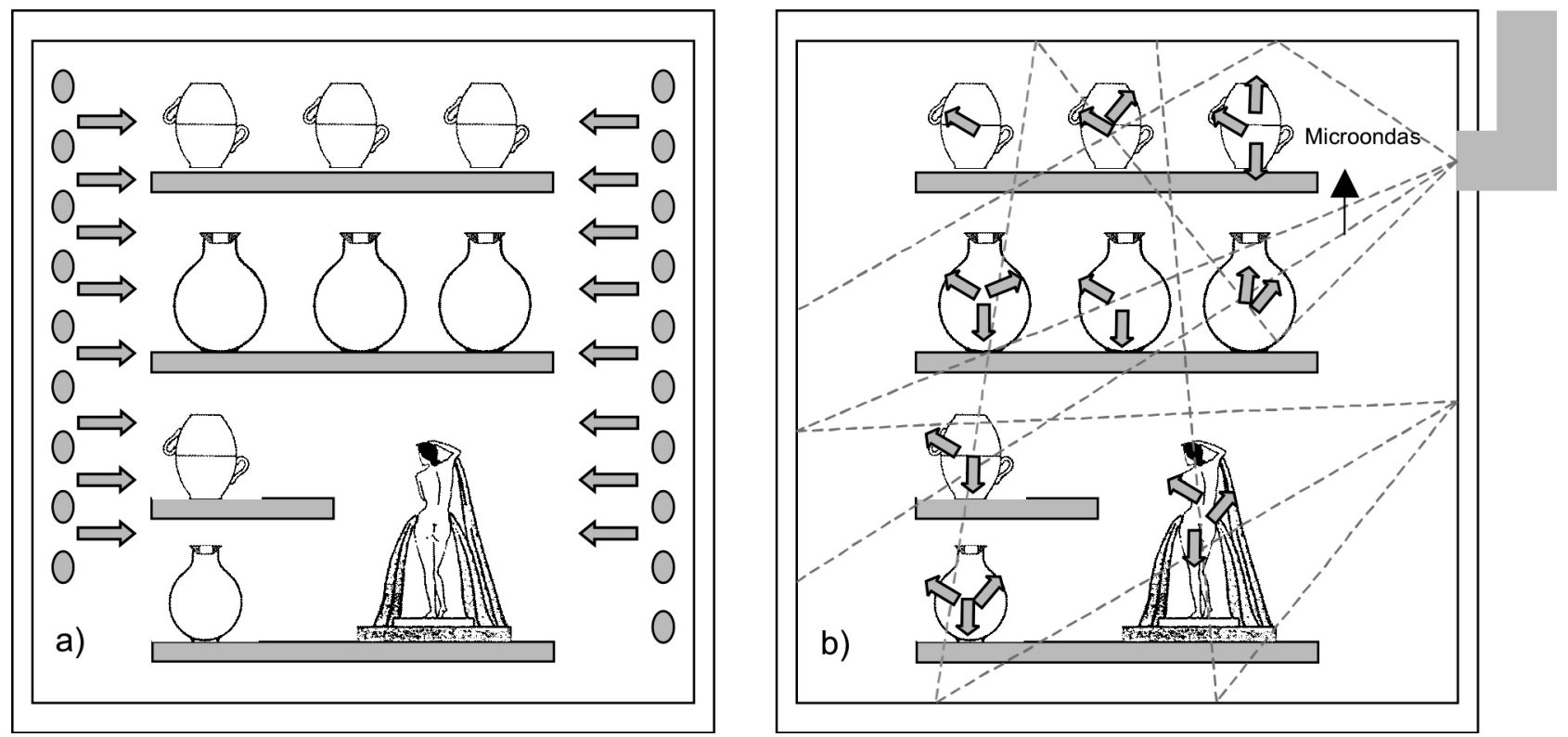

Figura 1: Padrão de aquecimento em a) forno convencional e b) forno de microondas.

[Figure 1: Heating pattern of a) conventional oven and b) microwave oven.] 
No processamento térmico convencional, ocorre, inicialmente, o aquecimento da superfície do material e só em seguida, através de processos de convecção e condução, a energia térmica é transferida para o interior do corpo cerâmico. Em contraste, a energia de microondas é transformada em calor diretamente dentro do material através da interação das moléculas e átomos com o campo eletromagnético, o que possibilita o aquecimento interno e volumétrico do material, provocando a formação de gradientes de temperaturas e fluxos de calor invertidos, comparativamente ao processo de aquecimento convencional [2]. Essas características permitem o aquecimento de peças grandes de maneira rápida e uniforme, sem a geração de elevadas tensões térmicas que podem causar trincas ou danos às peças. Como a absorção de energia de microondas varia com a composição e estrutura das fases, aquecimento seletivo também é possível. A Fig. 1 ilustra a diferença na forma de aquecimento do processamento convencional e daquele que usa microondas.

De forma geral, podem-se dividir as áreas de processamento de materiais cerâmicos com microondas em: recuperação (beneficiamento de minerais e reciclagem de resíduos); síntese (pirólise, combustão, decomposição, catálise); remoção de fases (secagem, volatilização de solventes, queima de orgânicos); fabricação (soldagem, CVI, cobertura - “coatings"); consolidação (secagem, sinterização, fusão, cura); processos de pós-fabricação (tratamento térmico, modificação superficial, nucleação/ cristalização) [3].

Os primeiros trabalhos abordando a utilização da energia de microondas no processamento cerâmico datam do início da década de 60, tendo conduzido a algumas aplicações comerciais. Estudos voltados ao processamento em elevadas temperaturas, tais como fusão e sinterização, começaram no final da década de 60 e início da década de 70. Todavia, muito da teoria sobre o comportamento dielétrico dos materiais e dados sobre a interação material/microondas, que forneceram uma grande base para o uso das microondas no processamento de materiais cerâmico, foram reportados já na década de 50 [4].

Durante a década de 70, houve um aumento no interesse pela utilização da energia de microondas em virtude da escassez de gás natural. Entretanto, apenas a partir do final da década de 80 , com os simpósios internacionais abordando o uso de microondas no processamento de materiais, que começaram a ser realizados a partir de 1988 e integraram a comunidade científica e industrial, ocorreu uma intensificação nos estudos voltados ao processamento de cerâmicas em microondas [3].

O grupo de pesquisas da Prof ${ }^{\mathrm{a}}$. Dr ${ }^{\mathrm{a}}$ Ruth Kiminami, LaDProM/DEMa/UFSCar foi um dos pioneiros no país a estudar o processamento de cerâmicas utilizando energia de microondas, tendo obtido vários sucessos na síntese de nanomateriais e compósitos, bem como na sinterização de cerâmicas [5-13].

Há um crescente interesse na utilização de energia de microondas no processamento cerâmico, especialmente em altas temperaturas (acima de $1500{ }^{\circ} \mathrm{C}$ ), em virtude do potencial para a diminuição nos custos de produção, com economia de energia e redução no tempo de processamento.

Atualmente, observa-se que a maioria dos estudos envolvendo processamento com microondas está direcionada a processos de síntese e/ou sinterização, visando à redução de tempo e/ou temperaturas de processo e ao processamento de nanomateriais [11-37]. Entretanto, um outro foco de pesquisas, que vem ganhando destaque, está relacionado ao estudo da influência da componente magnética do campo eletromagnético no processamento de metais e cerâmicas, e ao processamento de metais e carbetos metálicos [38-44]. O processamento de materiais metálicos entrou em evidência após os estudos desenvolvidos no final da década de 90 $[45,46]$, e apresenta interessantes perspectivas para o alargamento das aplicações da tecnologia de microondas.

Quanto à utilização comercial das tecnologias geradas nas últimas décadas, observa-se uma intensa movimentação nos últimos anos, tanto por parte dos pesquisadores quanto dos fabricantes de equipamentos, visando difundir, esclarecer e aplicar os conhecimentos e tecnologias desenvolvidos, nos processos industriais [43, 47-51]. Nesse sentido, já se observa aplicações em larga escala da tecnologia de microondas na secagem de materiais cerâmicos e no tratamento e vitrificação de resíduos.

\section{INTERAÇÃO MICROONDAS/MATERIAL}

Microondas são ondas eletromagnéticas que têm uma faixa de freqüência no espectro de energia de 0,3 a $300 \mathrm{GHz}$, com o correspondente intervalo de comprimento de onda de $1 \mathrm{~m}$ a $1 \mathrm{~mm}$. As ondas de microondas, tal como as ondas visíveis (luz visível), obedecem às leis da óptica e podem ser transmitidas, absorvidas ou refletidas dependendo do tipo de material. A Fig. 2 ilustra os tipos de interação das microondas com os materiais.

Através da Fig. 2, pode-se observar que existem basicamente três tipos de materiais, com relação à forma de interação com as microondas. Os materiais transparentes às microondas permitem a total passagem das ondas através de si, sem perdas significativas de energia. Um exemplo dessa categoria de materiais é a alumina, em temperatura ambiente. As cerâmicas são em geral transparentes às microondas; no entanto, quando aquecidas acima de uma determinada temperatura (temperatura crítica), passam a absorver mais eficientemente a radiação de microondas.

Os materiais opacos refletem as ondas eletromagnéticas, sendo os metais os principais representantes dessa categoria. A última categoria de materiais pode ser dividida em duas. A primeira subdivisão abrange os materiais com elevadas perdas dielétricas, que contêm uma ou mais fases. Nessa categoria estão, por exemplo, o $\mathrm{Co}_{2} \mathrm{O}_{3}, \mathrm{MnO}_{2}, \mathrm{NiO}, \mathrm{CuO}$, $\mathrm{BC}, \mathrm{SiC}$, etc. Esses materiais interagem com as microondas absorvendo-as eficientemente, o que provoca o seu rápido aquecimento. A segunda subdivisão é a categoria de materiais de absorção mista, em que a matriz é um isolante com baixas perdas, e a fase dispersa (ou fases) é constituída por 
materiais com elevadas perdas dielétricas. A adição de fases condutoras ou magnéticas possibilita aumentar a absorção de energia de microondas dos materiais "transparentes" à radiação de microondas. No compósito formado, as fases com altas perdas dielétricas irão aquecer preferencialmente, transferindo a energia para a matriz, o que permite que também aqueça rapidamente.

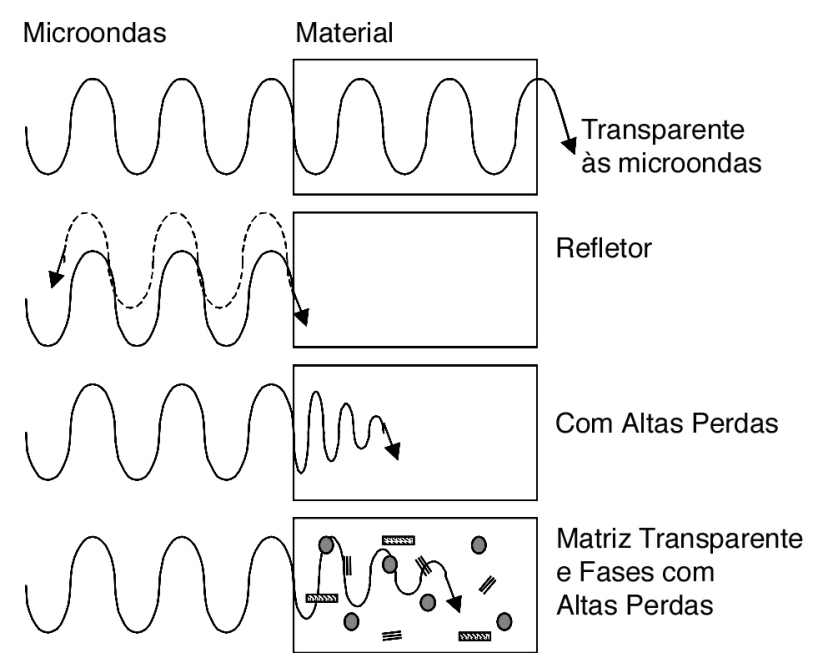

Figura 2: Interação das microondas com os materiais [4].

[Figure 2: Microwave/material interactions [4].]

O grau de interação (absorção) das ondas de microondas com um material dielétrico está relacionado com a sua permissividade complexa, $\varepsilon^{*}$ [4], que é composta por uma parte real, $\varepsilon^{\prime}$ (constante dielétrica) e por uma parte imaginária, $\varepsilon$ " (fator de perda dielétrica), sendo ambas função da freqüência da radiação:

$$
\varepsilon(\omega)^{*}=\varepsilon^{\prime}(\omega)-i \varepsilon ”(\omega)=\varepsilon_{0}\left(\varepsilon_{\mathrm{r}}{ }^{\prime}(\omega)-i \varepsilon_{\mathrm{r}}{ }^{\prime \prime}(\omega)\right)
$$

onde $i=(-1)^{1 / 2}, \varepsilon_{\mathrm{o}}$ é a permissividade no espaço $\left(\varepsilon_{0}=\right.$ $\left.8,86 \times 10^{-12} \mathrm{~F} / \mathrm{m}\right), \varepsilon_{\mathrm{r}}$ ' é a constante dielétrica relativa, $\varepsilon_{\mathrm{r}}$ " é o fator de perdas dielétricas relativo e $\omega$ é a freqüência angular.

A constante dielétrica é uma medida da quantidade de energia armazenada no material na forma de campo elétrico, enquanto que o fator de perdas é uma medida da energia dissipada na forma de calor dentro do material [52].

Quando as microondas penetram e se propagam através de um material dielétrico, o campo elétrico interno, gerado dentro do volume afetado, induz ao movimento translacional das cargas livres e ligadas ("bond charges"), como íons ou elétrons, e à rotação de cargas complexas, como os dipolos. A resistência a esses movimentos induzidos, devido à inércia elástica e forças de atrito, causa perdas e atenua o campo elétrico. Como conseqüência dessas perdas, ocorre o aquecimento do material [3].

Assim, de forma geral, tem-se que a radiação de microondas pode interagir com os materiais através de processos de polarização ou condução. A polarização envolve o deslocamento a curta distância das cargas, através da formação e rotação dos dipolos elétricos (ou dipolos magnéticos se estiverem presentes). A condução requer transporte de cargas de "longa" distância (comparativamente à rotação). Ambos os processos propiciam perdas em determinadas freqüências, e não é geralmente fácil diferenciar experimentalmente entre os dois mecanismos. Consequentemente, as perdas são tipicamente reportadas como perdas efetivas, $\varepsilon_{\text {eff }}$, quando os mecanismos de perdas são desconhecidos ou não podem ser claramente separados [4].

Como mencionado, os mecanismos de perdas são todos combinados juntos em um único fator de perdas, $\varepsilon_{\text {eff }}$, no entanto a tangente de perdas, tg $\delta$, é o parâmetro comumente utilizado para descrever essas perdas:

$$
\operatorname{tg} \delta=\frac{\varepsilon_{e f f}^{\prime \prime}}{\varepsilon^{\prime}}
$$

A tangente de perdas também pode ser referida como fator de dissipação. Todos os mecanismos de polarização são dependentes da freqüência e, por conseguinte, do tempo. Um dielétrico real, quando comparado com um dielétrico ideal, levará um certo tempo para responder ao estímulo do campo eletromagnético. A tangente de perdas indica o tempo de defasagem entre a aplicação do campo e a polarização do material. Este tempo provoca uma diferença de fase na corrente induzida no dielétrico, sendo essa diferença definida em termos do ângulo $\delta$. Assim, a tangente de perdas indica uma "perda" de energia devido ao tempo necessário para a polarização do material e também está relacionada com o armazenamento de carga [53].

O produto da freqüência angular e do fator de perdas é equivalente a uma condutividade dielétrica:

$$
\sigma=\omega \varepsilon^{\prime \prime}
$$

que engloba todos os efeitos de dissipação de energia [53].

A tangente de perdas e a constante dielétrica relativa são dois parâmetros que podem ser usados para determinar a potência absorvida, P, pelo material. Partindo-se de:

$$
P=\left.\int_{v} \sigma\left|E_{R u S}\right|_{\text {int }}\right|^{2} d v
$$

onde $\mathrm{E}_{\text {int }}$ é o campo elétrico interno, sendo dado em valor RMS ("root mean square") [54, 55], e utilizando-se a equação (C), tem-se:

$$
P=\left.\int_{v} \omega \varepsilon^{\prime \prime}\left|E_{R u S}\right|_{\text {int }}\right|^{2} d v
$$

Considerando-se que o campo eletromagnético está uniformemente distribuído dentro do material, pode-se fazer a seguinte simplificação:

$$
\frac{P}{V} \cong \frac{\omega \varepsilon^{\prime \prime} E_{\text {int }}{ }^{2}}{2}
$$


A equação (F) pode ser colocada em termo da tangente de perdas e da constante dielétrica, utilizando-se a equação (B) [3]:

$$
\frac{P}{V} \cong \frac{\omega \varepsilon^{\prime} \operatorname{tg} \delta E_{\text {int }}{ }^{2}}{2}
$$

Conforme as microondas penetram e se propagam dentro do material, há uma atenuação do campo elétrico. Essa atenuação do campo é descrita pela distância a partir da superfície onde o campo elétrico (considerando um material sem elevadas perdas magnéticas) é reduzido a 1/e do seu valor inicial. Esse parâmetro é conhecido por Profundidade de Penetração (ou Distância de Atenuação) e é dado por:

$$
D_{P}=\frac{\lambda_{o}}{2 \pi}\left[\frac{2}{\varepsilon_{r}^{\prime}\left(\sqrt{1+\operatorname{tg}^{2} \delta}-1\right)}\right]^{1 / 2}
$$

onde $\lambda_{\circ}$ é o comprimento de onda da radiação incidente.

Um outro parâmetro também importante no processamento com o uso de microondas é o coeficiente de reflexão, que é definido como a fração de potência refletida da onda eletromagnética incidente na superfície do material [56], o qual pode ser dado por:

$$
R=\frac{1-\sqrt{2 \varepsilon_{r}{ }_{r}\left[1+\sqrt{1+\operatorname{tg}^{2} \delta}\right.}+\varepsilon_{r}^{{ }_{r}} \sqrt{1+\operatorname{tg}^{2} \delta}}{1+\sqrt{2 \varepsilon^{\prime}{ }_{r}\left[1+\sqrt{1+\operatorname{tg}^{2} \delta}\right]}+\varepsilon^{{ }_{r}} \sqrt{1+\operatorname{tg}^{2} \delta}}
$$

As equações apresentadas permitem observar quais materiais são mais facilmente processados com a utilização de microondas. Materiais com elevada condutância e baixa capacitância, como os metais, possuem elevados fatores de perdas dielétricas, o que implica em profundidades de penetração próximas de zero. Materiais com esse comportamento são considerados refletores. Materiais com baixo fator de perdas dielétricas têm uma grande profundidade de penetração; entretanto, como conseqüência, muito pouco da energia é absorvido pelo material, que é considerado transparente à energia de microondas. Assim, materiais com perdas dielétricas com valores intermediários aos dois casos extremos são os mais facilmente processados com o uso de microondas.

Como mencionado anteriormente, a constante dielétrica, $\varepsilon^{\prime}$, e o fator de perdas, $\varepsilon$ ", são ambos função da freqüência da radiação. No entanto, esses parâmetros também variam com a temperatura do material. Como conseqüência, à medida que ocorre o aquecimento do material durante o seu processamento, ocorrem alterações nos valores de potência absorvida e refletida do material. As Fig. 3 e 4 ilustram as modificações da permissividade de amostra de $\mathrm{ZnO}$ e das tangentes de perdas de algumas aluminas, conforme se aumenta a temperatura.

Em geral, tem-se que $\varepsilon$ " é afetado mais intensamente pela temperatura que $\varepsilon^{\prime}$, o que é refletido na tangente de perdas. Inicialmente, a tangente aumenta de forma suave com a

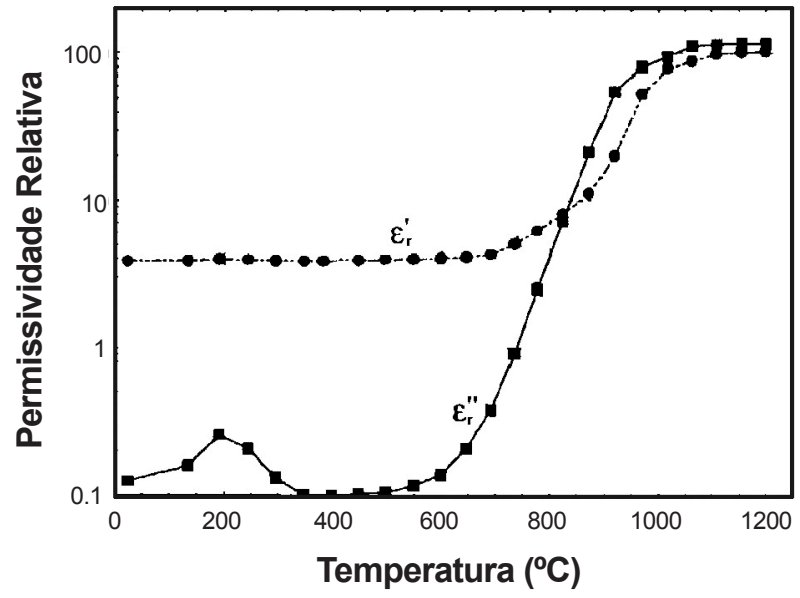

Figura 3: Comportamento da permissividade de amostra de $\mathrm{ZnO}$, em função da temperatura [57].

[Figure 3: Permissivity behaviour of $\mathrm{ZnO}$ sample in relation to temperature [57].]

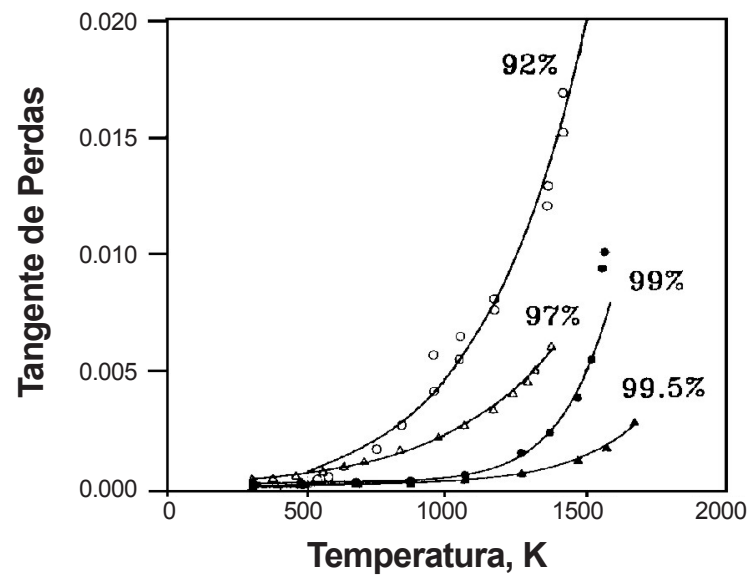

Figura 4: Comportamento da tangente de perdas de algumas amostras de alumina (\% indica a pureza) [58].

[Figure 4: Loss tangent behaviour of alumina samples (\% indicate purity) [58].]

elevação da temperatura, até uma temperatura crítica $\left(T_{\text {crit }}\right)$, a partir da qual, ocorre um aumento acentuado [3]. Adição de aditivos ou impurezas podem intensificar o aumento da $\operatorname{tg} \delta$ com a elevação da temperatura. Este comportamento é característico tanto de sólidos iônicos $\left(\mathrm{Al}_{2} \mathrm{O}_{3}, \mathrm{ZrO}_{2}\right)$, como de sólidos covalentes $\left(\mathrm{Si}_{3} \mathrm{~N}_{4}, \mathrm{AlN}\right)$, e ocorre, em temperaturas da ordem de 0,4 a 0,5 da temperatura de fusão. Nessa faixa de temperatura, as ligações entre íons, nos cristais iônicos, começam a ser rompidas e os elétrons, nos materiais covalentes, começam a povoar as bandas de condução, o que aumenta a condutividade do material. $\mathrm{O}$ amolecimento de fases vítreas e amorfas também é responsável por aumentos acentuados das perdas dielétricas dos materiais cerâmicos, sendo mais significativas em materiais com baixa pureza $(<98 \%)$ [59].

A Fig. 5 ilustra a alteração da profundidade de penetração, $\mathrm{D}_{\mathrm{p}}$, com o aumento da tangente de perdas, (calculados com a equação (H)) para alguns materiais, com base em valores de permissividade obtidos na literatura [60]. 
$T=20^{\circ} \mathrm{C}$

Alumina

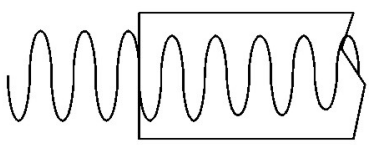

Zircônia

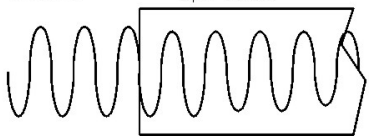

$\mathrm{T}=1200^{\circ} \mathrm{C}$
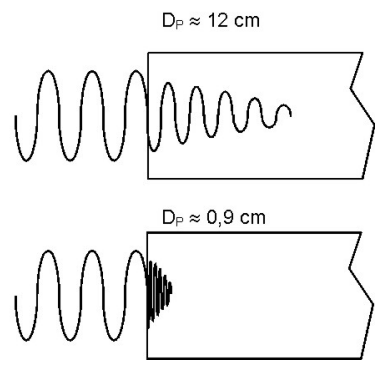

Figura 5: Profundidade de penetração $\left(\mathrm{D}_{\mathrm{p}}\right)$ de microondas em 2,45 GHz para alumina A16SG (densidade 98\%) e zircônia HSY-3 (densidade 98\%) [valores calculados a partir de dados de [60].

[Figure 5: Penetration deph $\left(D_{P}\right)$ at $2.45 \mathrm{GHz}$ to alumina A16SG (density of 98\%) and zirconia HSY-3 (density of 98\%) [values calculated using data from [60].]

Com o aumento da $\operatorname{tg} \delta$, o material absorve mais eficientemente a radiação de microondas, o que provoca a elevação da temperatura. $O$ aumento da temperatura faz com que a tg $\delta$ aumente de forma ainda mais rápida. Esse comportamento provoca o fenômeno denominado de "thermal runaway", que consiste no aumento exponencial da temperatura do material [3]. O "thermal runaway" é um aspecto muito importante no aquecimento por microondas, já que, se não for controlado, pode provocar um aquecimento indiscriminado do material, com a formação de pontos de superaquecimento ("hot spots"), fusão em regiões específicas ou em todo material, tensões devido aos gradientes de temperatura no material, etc. e não apenas aspectos positivos, como o aquecimento com taxas elevadas.

Nesse sentido, deve-se mencionar que a formação de "hot spots" está relacionada a heterogeneidades composicionais e/ou de densificação na amostra e a flutuações na intensidade do campo eletromagnético na câmara do forno, o que acarreta em heterogeneidades na sua distribuição dentro do corpo cerâmico. Isso faz, que partes da peça aqueçam de maneiras diferentes do restante.

\section{SINTERIZAÇÃO USANDO MICROONDAS}

Sinterizaçãopodeserdefinidacomoum tratamentotérmico para unir partículas, em uma estrutura predominantemente sólida, através de processos de transporte de massa, que ocorrem em escala atômica [61], que podem conduzir, ou não, à densificação do corpo.

Dificuldades envolvidas na densificação de materiais cerâmicos são oriundas da complexidade e grande número de mecanismos que estão envolvidos durante a sinterização. Pode-se analisar o processo de sinterização como uma competição entre os processos de difusão que favorecem a densificação e aqueles que provocam crescimento de grão, sendo necessário o ajuste das condições de processo para intensificar a densificação.

A sinterização rápida é uma técnica em que ocorre o rápido aquecimento das peças, seguido por sua sinterização em elevada temperatura, em curto período de tempo, podendo culminar em produtos de maior densidade relativa e menor tamanho de grão, comparativamente à sinterização convencional [62]. Isso está associado ao fato de se passar o menor tempo possível em baixas temperaturas, evitandose um crescimento de grão indesejável e, por conseguinte, não havendo a diminuição da curvatura da superfície das partículas (mantendo-se uma elevada força motriz para os processos de difusão que favorecerão à densificação), nem aumentos significativos da distância entre os contornos de grão e os poros, possibilitando que em altas temperaturas, se obtenham elevadas densificações.

A sinterização rápida convencional, apesar de conduzir a elevadas densificações em curto período de tempo, se depara com obstáculos que limitam seu uso. Dentre eles, tem-se o desenvolvimento de tensões térmicas na peça, oriundas de gradientes de temperatura presentes no processo de sinterização. Essas tensões podem conduzir à formação de trincas e deterioração das propriedades mecânicas do material. Também como conseqüência de gradientes térmicos, pode ocorrer o crescimento irregular dos grãos ao longo da peça, gerando heterogeneidade microestrutural.

$\mathrm{O}$ aquecimento por meio de microondas é uma forma de processamento, que, em virtude de características particulares, possibilita ultrapassar as limitações do processo de queima rápida convencional. Essas características são oriundas dos mecanismos de geração de calor envolvidos na utilização das microondas, que permitem um aquecimento volumétrico da peça, de forma rápida e mais uniforme que no caso da sinterização convencional [1, 4]. Entretanto, deve-se salientar que estudos evidenciaram que, se forem utilizadas baixas taxas de aquecimento no processamento por microondas, o gradiente de temperatura desenvolvido na amostra pode vir a ser maior que o obtido com o aquecimento convencional.

Além do aquecimento volumétrico, há uma outra característica que sugere que a sinterização por microondas se diferencia significativamente da convencional, que é a intensificação das taxas de densificação. Na maioria dos trabalhos abordando processamento com a utilização de microondas, os autores reportam algum tipo de aceleração no processo. A aceleração comumente manifesta-se através da redução no tempo e/ou temperatura de sinterização, comparativamente ao processamento convencional, ou com maiores taxas de reação e/ou cinéticas de reações significativamente diferentes das observadas no processamento convencional [2]. Este fato é conhecido como "efeito microondas" ("microwave effect"), ou mais estritamente falando, "efeito não-térmico provocado pelo campo eletromagnético" e é observado em praticamente todas as áreas de aplicação de microondas [4].

Nos processos de sinterização, o "efeito microondas" é geralmente quantificado pela diferença entre as temperaturas nos dois tratamentos $-\Delta \mathrm{T}=\mathrm{T}_{\text {convencional }}-\mathrm{T}_{\text {microondas }}-$, que levam os corpos cerâmicos à mesma densidade. Como exemplo, têm-se dados [63] que reportam $\Delta$ Ts de 300 a $400{ }^{\circ} \mathrm{C}$ para a sinterização de alumina a $28 \mathrm{GHz}$. No entanto, outros estudos 
[64] advogam $\Delta$ Ts mais modestos, de 50 a $300^{\circ} \mathrm{C}$, o que está muito associado à freqüência utilizada no processamento, ao tipo de material processado e às particularidades de aquecimento. Por outro lado, críticos ao "efeito microondas" acreditam que as diferenças de temperatura estão relacionadas a medidas inadequadas e à falta de controle das condições experimentais, o que conduziria a erros sistemáticos nos valores medidos de temperaturas [1].

Os menores tempos e/ou temperaturas de processamento se refletem em redução nos custos energéticos do processo. Foi indicado [65] que a energia específica consumida no processo de sinterização de cerâmicas à base de alumina (na freqüência de $2,45 \mathrm{GHz}$ ), em temperatura da ordem de $1600{ }^{\circ} \mathrm{C}$, está em torno de $4 \mathrm{kWh} \mathrm{kg}^{-1}$ para o aquecimento por microondas versus $59 \mathrm{kWh} \mathrm{kg}^{-1}$ para a sinterização rápida convencional em forno de resistência. Outros autores [49] evidenciaram reduções mais modestas, de $7,5 \mathrm{kWh} \mathrm{kg}^{-1}$, para $2,5 \mathrm{kWh} \mathrm{kg}^{-1}$. Também foi observado, para a sinterização de cerâmicas à base de nitreto de silício, que o consumo específico de energia está em torno de $3 \mathrm{kWh} \mathrm{kg}^{-1} \mathrm{em}$ um ciclo de $2 \mathrm{~h}$, ao se processar utilizando microondas $(2,45 \mathrm{GHz})$, enquanto está em torno de $20 \mathrm{kWh} \mathrm{kg}^{-1}$ em um ciclo de $12 \mathrm{~h}$ no processo convencional [66]. Comparativamente à prensagem a quente, observou-se [67] que na sinterização do carbeto de boro é possível uma redução de $18 \%$ no gasto energético.

A utilização de microondas como um método de processamento em elevadas temperaturas possui muitos benefícios. Todavia, dificuldades podem surgir caso o material a ser processado não absorva adequadamente as microondas em baixas temperaturas, como é o caso da maioria das cerâmicas, quando processadas utilizando-se baixas freqüências (inferiores a 2,45 GHz). Nesses casos, longos tempos são requeridos para o aquecimento do material, o que acarreta uma série de pontos negativos ao processamento, como gradientes de temperatura na amostra, crescimento exagerado de grãos e heterogeneidades microestruturais $[59,68]$. Isso desfaz os pontos positivos da utilização da energia de microondas.

Os problemas com a sinterização podem se intensificar, caso sejam utilizadas altas potências de irradiação, objetivando contrapor as baixas perdas dielétricas do material, o que pode provocar gradientes de temperatura mais acentuados, excitação dos gases presentes na câmara do forno, com formação de descargas elétricas, "thermal runaway" descontrolado, geração de trincas e até destruição da amostra [67, 69-71].

Para superar essas dificuldades, obtendo-se um aquecimento rápido em baixas temperaturas e uniformidade na distribuição de temperaturas no corpo cerâmico durante a sinterização, foi desenvolvida a sinterização híbrida. A qual possibilita obter microestruturas uniformes em ciclos de aquecimento rápidos e controlados, mesmo trabalhando-se com materiais com baixas perdas dielétricas na temperatura ambiente e na freqüência de $2,45 \mathrm{GHz}$.

A sinterização híbrida com microondas (do inglês "microwave hybrid heating - MHH"), também conhecida como sinterização assistida, é usada para processar materiais que são transparentes à energia de microondas em baixas temperaturas. O aquecimento híbrido possibilita, também, uma distribuição de temperatura uniforme no corpo cerâmico, durante sua sinterização em altas temperaturas, independentemente da necessidade ou não de auxílio para o aquecimento inicial do material em baixas temperaturas. Deve-se ressaltar que a utilização de aquecimento híbrido não ocorre apenas em sinterização, mas em vários processos em diversas áreas, como na indústria alimentícia, na síntese de materiais cerâmicos, na área de catálise, etc [52].

A utilização de microondas como um método de processamento em elevadas temperaturas possui muitos benefícios; todavia, como mencionado anteriormente, dificuldades podem surgir caso o material a ser processado não absorva adequadamente microondas em baixas temperaturas, como é o caso da maioria das cerâmicas.

Para transpor essas dificuldades, dois caminhos podem ser adotados: pode-se alterar a freqüência da radiação de microondas incidente, para uma freqüência na qual o material absorva a radiação mais eficientemente, e aqueça; ou pode-se utilizar uma forma de aquecimento indireto até determinada temperatura, a partir da qual, o material interagindo eficientemente com as microondas aquecerá, já que, em elevadas temperaturas, a absorção de microondas na maioria dos materiais aumenta intensamente. Essa intensificação na absorção de microondas se dá em temperaturas da ordem de $0,4-0,5 \mathrm{~T}_{\mathrm{m}}\left(\mathrm{T}_{\mathrm{m}}-\right.$ temperatura de fusão) e está muito relacionada à pureza do material.

No primeiro caso, tem-se que a freqüência ótima para o aquecimento irá variar de acordo com o material em questão. $\mathrm{O}$ uso de freqüências específicas é geralmente difícil de ser realizado na prática, em virtude de só haver equipamentos disponíveis comercialmente operando nas freqüências de $912 \mathrm{MHz}$ e 2,45 GHz. Ademais, se a freqüência de processamento ótima varia de acordo com o material, seria necessária a construção de um equipamento para cada freqüência caso se deseje processar vários materiais, o que é praticamente impensável.

Já existem equipamentos que permitem a utilização de várias freqüências ou faixas de freqüências, bem como sua mudança durante o processo; no entanto, seu custo é excepcionalmente elevado [72].

A segunda opção abrange duas possibilidades: o uso de um sistema externo de aquecimento convencional ou o uso de um material adicional na câmara do forno, que absorve eficientemente a energia eletromagnética e aquece a amostra. Esse material adicional é chamado de susceptor. Essa segunda opção recebe o nome de aquecimento híbrido.

$\mathrm{O}$ aquecimento híbrido realizado com o uso de um sistema de aquecimento externo, utiliza-se de arranjos de aquecimento convencionais, como resistências elétricas, sistemas de indução, queima de gás natural, etc. em parceria com a energia de microondas. A utilização de sistemas de aquecimento externo está sendo muito estudada para uso em fornos industriais, principalmente com queima de gás natural. O sistema híbrido microondas/gás é o que apresenta maior eficiência energética [4]; entretanto, resultado recente 
[49] indica que o processamento em microondas de peças maiores (acima de $300 \mathrm{~g}$ ) usando susceptores, apresenta um custo equivalente ao sistema microondas/gás. A interferência do sistema de aquecimento com gás sobre a distribuição do campo eletromagnético no forno de microondas e sua influência na atmosfera de queima ainda não foram detalhadamente estudadas. Bem como a forma de controle de aquecimento, a fim de se ter balanço otimizado entre a energia aplicada com as microondas e a oriunda da queima do gás.

Quando se usa um material susceptor, esse irá aquecer a amostra (peça) em baixas temperaturas e na freqüência em que o equipamento é comercialmente disponível. Após atingir uma temperatura mais elevada (que varia de material para material), a peça passa a absorver a energia de microondas mais eficientemente e aquece rapidamente.

Nesse sentido há dois tipos de susceptores: os consumidos durante a sinterização e os permanentes. Os que são consumidos abrangem carbono, feltros de carbono, ligantes, compostos químicos introduzidos como aditivos ou exclusivamente para aquecimento (como nitratos e uma série de polímeros), etc., que queimam em elevadas temperaturas. Esse tipo de susceptor serve apenas para aquecer a amostra até determinada temperatura, a partir da qual ela possa absorver eficientemente as microondas. O uso de susceptores que queimam ou se decompõem durante o processo pode gerar gradientes invertidos de temperatura acentuados na amostra e induzir a formação de heterogeneidades microestruturais.

Outro ponto importante é o controle da taxa de combustão dos orgânicos, em virtude da pressão interna gerada com a formação dos gases de queima, bem como da atmosfera presente na câmara, a fim de garantir que ocorrerá a queima do material e não ficarão resíduos dos aditivos na amostra $[73,74]$.

Os susceptores permanentes não queimam em altas temperaturas, permanecendo "intactos" durante todo o processo de queima. O carbeto de silício, por possuir elevada resistência química ao ataque de uma grande quantidade de gases e fluidos, possuir elevadas perdas dielétricas e elevada estabilidade estrutural em altas temperaturas é o material mais utilizado como susceptor [72]. Esse tipo de susceptor absorve a radiação de microondas e aquece a peça para que atinja sua temperatura crítica. Em altas temperaturas passam a existir duas fontes de aquecimento para a peça:

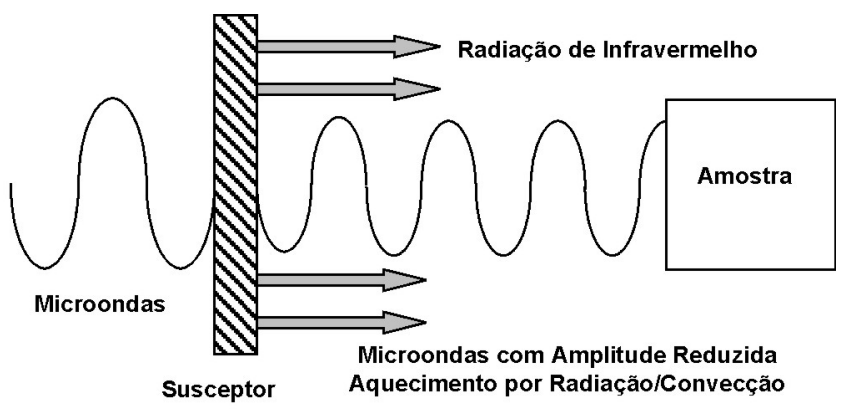

Figura 6: Aquecimento com a utilização de susceptor permanente. [Figure 6: Hybrid heating using a non-consumable susceptor.] o aquecimento volumétrico por meio das microondas e o calor fornecido (radiação de infravermelho e correntes de convecção, ou condução) pelo susceptor, obtendo-se uma distribuição mais uniforme de temperatura. A qual reduz as tensões térmicas do processo de sinterização, bem como produz uma microestrutura mais uniforme. A Fig. 6 ilustra o processo de aquecimento com o uso de susceptores.

Assim, com base no exposto pode-se observar que o conhecimento de alguns parâmetros e peculiaridades do processamento de materiais utilizando microondas são fundamentais para obter êxito na aplicação de microondas na sinterização de cerâmicas. Verifica-se também, que as dificuldades e obstáculos que podem surgir no processo de sinterização de cerâmicas utilizando-se microondas podem ser superados tendo-se o entendimento científico de suas causas. $\mathrm{O}$ que permite que sejam adotados, de forma rápida e eficiente, os procedimentos e alternativas mais adequados para a solução dessas dificuldades, tal como a sinterização híbrida. Nesse sentido os próximos trabalhos, Sinterização de Cerâmicas em Microondas Parte II e III, abordam a sinterização híbrida com microondas de uma série de cerâmicas, evidenciando a eficiência da técnica para a sinterização rápida de cerâmicas com os mais diversos propriedades e comportamentos dielétricos tanto em baixas $\left(<1200^{\circ} \mathrm{C}\right)$ como em altas temperaturas.

\section{AGRADECIMENTOS}

FAPESP (05/60095-6, 06/50121-5) e ao CNPq (476382/ 2004-1) pelo apoio financeiro.

\section{REFERÊNCIAS}

[1] E. T. Thostenson, T. W. Chou, Composites: Part A 30 (1999) 1055.

[2] D. K. Agrawal, Current Opinion in Solid State \& Mater. Sci. 3 (1998) 480.

[3] D. E. Clark, W. H. Sutton, Ann. Rev. Mater. Sci. 26 (1996) 299.

[4] W. H. Sutton, Am. Ceram. Soc. Bull. 68, 2 (1989) 376.

[5] R. H. G. A. Kiminami, M. R. Morelli, D. Folz, D. E. Clark, Am. Ceram. Soc. Bull. 79, 3 (2000) 63.

[6] R. H. G. A. Kiminami I, M. R. Morelli, D. Folz, D. E. Clark, Metall. and Mater. Trans. A 42, 8 (2001) 1661.

[7] E. Fagury Neto, R. H. G. A. Kiminami, Ceram. Int. 27, 7 (2001) 815.

[8] R. H. G. A. Kiminami, M. R. Morelli, D. Folz, D. E. Clark, Ceram. Trans., Microwaves: Theory and application in materials processing V, Eds: D. E. Clark, D. A. Lewis, Am. Ceram. Soc., Westville, OH, 111 (2001) 189.

[9] E. Fagury Neto, R. H. G. A. Kiminami, Mater. Sci. Forum 416 (2003) 693.

[10] A. C. Costa, E. Fagury Neto, R. H. G. A. Kiminami, Mater. Sci. Forum 416 (2003) 705.

[11] C. C. Paula, R. R. Menezes, D. Garcia, J. Eiras, R. H. G. A. Kiminami, Proc. $4^{\text {th }}$ World Cong. Microwave and Radio Frequency Appl., Eds: D. Folz, W. Shulz, Microwave 
Working Group, Arnold (2005) 142.

[12] R. R. Menezes, P. M. Souto, E. Fagury Neto, R. H. A. Kiminami, Proc. $4^{\text {th }}$ World Cong. Microwave and Radio Frequency Appl., Eds: D. Folz, W. Shulz, Microwave Working Group, Arnold (2005) 118.

[13] T. P. Deksnys, R. R. Menezes, E. Fagury Neto, R. H. G. A. Kiminami, Ceram. Int. 33, 1 (2007) 67.

[14] A. Goldstein, R. Ruginets, Y. Geffen, J. Mater. Sci. Lett. 16 (1997) 310.

[15] Z. S. Ahn, H. B. Lee, J. Mater. Sci. 33 (1998) 4255.

[16] S. A. Suvorov, I. A. Turkin, L. N. Printsev, Refractories and Industrial Ceramics 41, 11-12 (2000) 417.

[17] W. C., Lee, K. S., Liu, I. N. Lin, J. Mater. Sci. 25 (2000) 4841.

[18] Z. Xie, Z. Gui, L. Li, X. Huang, Y. Huang, J. Mater. Sci. 35 (2000) 203.

[19] D. D. Upadhyaya, A. Ghosh, G. K. Dey, R. Prasad, A. K. Suri, J. Mater. Sci. 36 (2001) 4707.

[20] H. Katsuki, S. Furuta, S. Komarneni, J. Porous Mater. 8 (2001) 5.

[21] E. M. Dedlovskaya, N. P. Kuz'mina, A. B. Antipov, A. N. Grigor'ev, L. I. Martynenko, Inorganic Materials 38, 12 (2002) 1277.

[22] S. Marinel, M. Pollet, G. Desgardin, J. Mater. Sci.: Materials in Electronics 13 (2002) 149.

[23] W. Yang, A. Chang, B. Yang, J. Mater. Synth. Proc. 10, 6 (2002) 303.

[24] J. Cheng, D. Agrawal, Y. Zhang, R. Roy, J. Electroceram. 9 (2002) 67.

[25] A. Bhaskar, B. Rajini, S. R. Murthy, J. Mater. Sci. 39 (2004) 3787.

[26] A. Chekanova, E. A. Eremina, A. S. Vanetsev, Y. D. Tret'yakov, Inorganic Materials 40 (2004) 420.

[27] I. Gómez, M. Hernández, J. Aguilar, M. Hinojosa, Ceram. Int. 30 (2004) 893.

[28] B. Vaidhyanathan, D. K. Agrawal, R. Roy, J. Am. Ceram. Soc. 87, 5 (2004) 834.

[29] P. Ramesh, A. Badzian, R. Roy, J. Am. Ceram. Soc. 87, 9 (2004) 1806.

[30] Y. J. Yang, C. L. Sheu, S. Y. Cheng, H. Y. Chang, J. Magn. Magn. Mater. 284 (2004) 220.

[31] A. B. Corradi, F. Bondioli, B. Focher, A. M. Ferrari, C. Grippo, E. Mariani, C. Villa, J. Am. Ceram. Soc. 88, 9 (2005) 2639.

[32] A. V. Orlov, A. S. Vanetsev, A. A. Kovalenko, O. A. Lyapina, Y. D. Tret'yakov, Doklady Chemistry 403 (2005) 118.

[33] J. Binner, Industrial Heating 72, 6 (2005), 43.

[34] K. S. Kumar, T. Mathew, J. Alloys Comp. 391 (2005) 177.

[35] S. Baldassari, S. Komarneniw, E. Mariani, C. Villa, J. Am. Ceram. Soc. 88, 11 (2005) 3238.

[36] D. Li, S. Komarneniw, J. Am. Ceram. Soc. 89, 5 (2006) 1510.

[37] N. Azurmendi, I. Caro, A. C. Caballero, T. Jardiel, M. Villegasw, J. Am. Ceram. Soc. 89, 4 (2006) 1232.

[38] J. Cheng, R. Roy, D. K. Agrawal, J. Mater. Sci. Lett. 20
(2001) 1561.

[39] J. Cheng, R. Roy, D. K. Agrawal, Mater. Res. Innov. 5 (2002) 170 .

[40] D. C. Dube, P. D. Ramesh, J. Cheng, M. T. Lanagan, D. Agrawal, R. Roy, Appl. Phys. Lett. 85, 16 (2004) 3632.

[41] J. Lu, Appl. Phys. Lett. 84, 25 (2004) 5076.

[42] J. Lu, Int. J. Infrared and Millimeter Waves 25, 9 (2004) 1271.

[43] E. B. Ripley, J. A. Oberhaus, Industrial Heating 72, 5 (2005) 65.

[44] R. Roy, Y. Fang, J. Cheng, D. K. Agrawal, J. Am. Ceram. Soc. 88, 6 (2005) 1640.

[45] K. Rijdiger, M. W. Porada, Int. J. Refractory Metals \& Hard Mater. 16 (1998) 409.

[46] R. Roy, Nature 399 (1999) 668.

[47] A. Hajduk, Ceramic Industry 153, 5 (2003) 32.

[48] M. Krzysica, Ceramic Industry 153, 5 (2003) 25.

[49] H. Shulman, Industrial Heating 70, 3 (2003) 59.

[50] D. K. Agrawal, Industrial Heating 72, 6 (2005) 37.

[51] C. L. Grahl, Ceramic Industry 154, 4 (2005) 43.

[52] B. Vos, J. Mosman, Y. Zhang, E. Poelsa, A. Bliek, J. Mater. Sci. 38 (2003) 173.

[53] L. L. Hench, J. K. West, Principles of electronic ceramics, John Wiley \& Sons, New York, USA (1990) 234. [54] R. W. Bruce, Materials Research Society Symp. Proc.: Microwave processing of materials I, Eds.: W. Sutton, M. H. Brooks, I. J. Chabinsky, Materials Research Society, Pittsburgh, 124 (1988) p. 3.

[55] W. R. Tinga, Materials Research Society Symp. Proc.: Microwave processing of materials I, Eds.: W. Sutton, M. H. Brooks, I. J. Chabinsky, Materials Research Society, Pittsburgh, 124 (1988) 33.

[56] M. Abraham, R. Becker, The classical theory of electricity and magnetism, $2^{\text {nd }}$ Ed., Blackie \& Son, London (1952) 288.

[57] A. Birnboim,. D. Gershon, J. Calame, A. Birman, Y. Carmel, J. Rodgers, B. Levush, Y. V. Bykov, A. G. Eremeev, V. V. Holoptsev, V. E. Semenov, D. Dadon, P. L. Martin, M. Rosen, R. Hutcheon, J. Am. Ceram. Soc. 81, 6 (1998) 1493. [58] M. S. Spotz, D. J. Skamser, D. L. Johnson, J. Am. Ceram. Soc. 78, 4 (1995) 1041.

[59] W. W. Ho, Materials Research Society Symp. Proc.: Microwave processing of materials I, Eds.: W. Sutton, M. H. Brooks, I. J. Chabinsky, Materials Research Society, Pittsburgh, 124 (1988) 137.

[60] M. Arai, Ceram. Trans., Microwaves: Theory and applications in materials processing II, Eds: J. Binner, D. Whitaker, Am. Ceram. Soc., Westerville, OH, 36 (1993) 483. [61] R. German, Sintering theory and practice, John Wiley \& Sons, New York (1996) 548.

[62] M. Harmer, R. J. Brook, J. Brit. Ceram. Soc. 80, 5 (1981) 147.

[63] M. A. Janney, H. D. Kimrey, Ceram. Trans., Sintering of advanced ceramics, Am. Ceram. Soc., Westerville, OH, 7 (1990) 382.

[64] Ph. Boch; N. Lequex, Solid State Ionics 101-103 (1997) 1229. 
[65] M. C. L. Patterson, M. C. L. Kimber, APTE, P. S., Materials Research Society Symposium Proc., Microwave processing of materials II, Eds.: W. B. Synder, W. H. Sutton, D. L. Johnson, M. F. Iskander, Materials Research Society, Pittsburgh, 189 (1990) 257.

[66] M. C. L. Patterson, Materials Research Society Symposium Proc., Microwave processing of materials III. Eds.: R. L. Beatty, W. H. Sutton, M. F. Iskander, Materials Research Society, Pittsburgh, 269 (1992) 291.

[67] J. D. Katz, Materials Research Society Symposium Proc., Microwave processing of materials I, Eds.: W. Sutton, M. H. Brooks, I. J. Chabinsky, Materials Research Society, Pittsburgh, Vol. 124 (1988) 219.

[68] D. S. Patil, Ceram. Trans., Microwaves: Theory and applications in materials processing, Eds.: D. E. Clark, F. D. Gac, W. H. Sutton, Am. Ceram. Soc., Westerville, OH, 21 (1991) 301.

[69] M. A. Janney, C. L. Calhon, H. D. Kimrey, Ceram. Trans., Microwaves: Theory and applications in materials processing, Eds.: D. E. Clark, F. D. Gac, W. H. Sutton, Am.
Ceram. Soc., Westerville, OH, 21 (1991) 311.

[70] Y. L Tian, Ceram. Trans., Microwaves: Theory and applications in materials processing, Eds.: D. E. Clark, F. D. Gac, W. H. Sutton, Am. Ceram. Soc., Westerville, OH, 21 (1991) 283.

[71] M. A. Janney, C. L. Calhon, H. D. Kimrey, J. Am. Ceram. Soc. 75, 2 (1992) 341.

[72] K. S. Leiser, D. E. Clark, Ceram. Trans., Microwaves: Theory and application in materials processing V, Eds: D. E. Clark, D. A. Lewis, Am. Ceram. Soc., Westville, OH, 111 (2001) 267.

[73] G. V. A. Tayler, M. Hamlyn, M. Anderson, Ceramic transactions, Microwaves: Theory and applications in materials processing IV, Eds.: D. E. Clark, W. H. Sutton, D. A. Lewis, Am. Ceram. Soc., Westerville, OH, 80 (1997) 547.

[74] G. V. A. Tayler, M. Anderson, Ceramic transaction, Microwaves: Theory and application in materials processing V, Eds: D. E. Clark, D. A. Lewis, Am. Ceram. Soc., Westville, OH, 111 (2001) 295.

(Rec. 01/09/2006, Ac. 24/11/2006) 